\title{
Genetic determinants of right-ventricular remodeling after tetralogy of Fallot repair
}

\author{
Aamir Jeewa' ', Ashok Kumar Manickaraj', Luc Mertens' ', Cedric Manlhiot' ', Caroline Kinnear', Tapas Mondal', John Smythe ,
} Herschel Rosenberg ${ }^{4}$, Jane Lougheed ${ }^{5}$, Brian W. McCrindle' ${ }^{1}$, Glen van Arsdell' ${ }^{1}$ Andrew N. Redington' ${ }^{1}$ and Seema Mital'

BACKGROUND: Hypoxia-inducible factor (HIFTA) regulates the myocardial response to hypoxia and hemodynamic load. We investigated the association of HIF 1 A variants with right-ventricular (RV) remodeling after tetralogy of Fallot (TOF) repair.

METHODS: Children with TOF were genotyped for three single-nucleotide polymorphisms in HIFIA. Genotypes were analyzed for association with RV myocardial protein expression and fibrosis at complete repair $(n=42)$ and RV dilation, fractional area change, and freedom from pulmonary valve/conduit replacement on follow-up.

RESULTS: In 180 TOF patients, mean age at repair was $1.0 \pm$ $0.8 \mathrm{y}$ with follow-up at $9.0 \pm 3.5 \mathrm{y} ; 82 \%$ had moderate to severe pulmonary insufficiency. Freedom from RV reinterventions at 5, 10, and 15 y was 92,84 , and 67\%, respectively. Patients with more functioning HIFIA alleles had higher transforming growth factor $\beta 1$ expression and more fibrosis at initial repair as compared with controls $(P<0.05)$. During follow-up, patients with more functioning HIFIA alleles showed less RV dilation, better preservation of RV function, and greater freedom from $\mathrm{RV}$ reinterventions $(P<0.05)$. This was confirmed in a replication cohort of 69 patients.

CONCLUSION: In children who have had TOF repair, a lower number of functioning HIF1A alleles was associated with RV dilation and dysfunction, suggesting that hypoxia adaptation in unrepaired TOF may influence RV phenotype after repair.

$\mathrm{T}$ etralogy of Fallot (TOF) is the most common cyanotic congenital heart defect, occurring in 4 of every 10,000 births (1). "Classic" TOF comprises pulmonary stenosis, ventricular septal defect, aortic override, and right-ventricular (RV) hypertrophy. In the unrepaired state, the right ventricle is exposed to hypoxia and pressure overload. Even after complete surgical repair (typically performed in early infancy), persistent pulmonary regurgitation after repair is common and contributes to progressive RV dilation often culminating in RV failure, ventricular arrhythmias, and reduced survival (2). Recent attempts at preserving pulmonary valve function at primary repair have not translated into long-term benefits on RV dilation and dysfunction $(3,4)$. Similarly, pulmonary valve replacement after the onset of RV dilation facilitates reverse
RV remodeling but does not restore normal ventricular function and exercise capacity (5). Therefore, the strategy of relying on RV dilation as a primary indication for reintervention has met with limited success. In order to identify at-risk patients early, before significant RV modeling has occurred, requires a better understanding of the genes and biologic pathways that mediate RV remodeling.

One such pathway is the hypoxia-response pathway, which is activated in response to both hypoxia and mechanical or hemodynamic stress. The right ventricle is exposed to chronic hypoxia and pressure overload during fetal life and infancy before TOF repair, and to ongoing hemodynamic stresses related to volume overload from pulmonary insufficiency and/or pressure overload from conduit stenosis after TOF repair. A central mediator of cardiac adaptation to hypoxia and mechanical stress is hypoxia-inducible factor 1 a (HIF1A). In acute hypoxia, HIF1A is cardioprotective by virtue of its ability to induce angiogenic, metabolic, and erythropoietic genes $(6,7)$. Chronic HIF1A upregulation however can promote transforming growth factor $\beta 1$ (TGF $\beta 1$ )-mediated organ fibrosis $(8,9)$. TGF $\beta 1$ promotes the transformation of endothelial and smooth muscle cells to fibroblasts. With ongoing stress, fibroblasts convert to proliferating myofibroblasts that induce extracellular matrix deposition and fibrosis. This mechanism for cardiac fibrosis was first reported by Zeisberg et al. (9) in mouse hearts in response to pressure overload. Importantly, this process of fibroblast transformation was reversible by TGF $\beta 1$ inhibition. The process of active fibroblast transformation may therefore reflect a reversible protective response to environmental stress that facilitates the transition of stressed endothelial or smooth muscle cells into fibroblasts to help them survive in adverse environmental conditions. These processes have not been evaluated in TOF, in which the right ventricle is exposed to chronic hypoxia and hemodynamic stresses. Our previous study showed that several TOF patients with severe hypoxia showed impaired RV adaptation, with downregulation of vascular endothelial growth factor (VEGF) and antioxidant enzymes and upregulation of collagen and plasma lactate levels postoperatively (10). However, the cause of

\footnotetext{
'Department of Pediatrics, Hospital for Sick Children, Toronto, Ontario, Canada; ${ }^{2}$ Department of Pediatrics, Hamilton Health Sciences Centre, Hamilton, Ontario, Canada; ${ }^{3}$ Department of Pediatrics, Kingston General Hospital, Kingston, Ontario, Canada; ${ }^{4}$ Department of Pediatrics, London Health Sciences Centre, London, Ontario, Canada; ${ }^{5}$ Department of Pediatrics, Children's Hospital of Eastern Ontario, Ottawa, Ontario, Canada. Correspondence: Seema Mital (seema.mital@sickkids.ca)
} 
impaired myocardial adaptation to hypoxia in some patients and the long-term impact of chronic hypoxia on RV remodeling were not studied.

The purpose of this study was to evaluate the influence of genetic variations in HIF1A on RV angiogenic factor expression and fibrosis at the time of surgical repair in infancy and on RV remodeling during serial follow-up after surgery. Specifically, we investigated the association of three common variants in the HIF1A gene with RV fibrosis at the time of complete repair and on RV size, RV function, and freedom for RV reinterventions during follow-up.

\section{RESULTS}

\section{Primary Cohort}

One hundred and eighty eligible patients were included; 55\% were male. Clinical and echocardiographic characteristics are described in Table 1 . Median age at repair was $0.6 \mathrm{y}$, mean age at follow-up was $9 \mathrm{y}, 84 \%$ had "classic" TOF, and $82 \%$ had moderate to severe pulmonary regurgitation at last follow-up.

\section{HIF1A Allele Frequencies}

Frequencies of functioning $H I F 1 A$ alleles were: HIF1A145T 74\%, HIF1A1326C 82\%, and HIF1A1744C 89\%. Cumulative frequencies were as follows: $1 \%$ of the cohort had one, $2 \%$ had two, $16 \%$ had three, $15 \%$ had four, $14 \%$ had five, and $51 \%$ had six functioning alleles. Genotype frequencies were comparable with the ventricular septal defects controls as well as the general population (data not shown). All genotypes were in Hardy-Weinberg equilibrium with no gender-based differences in single-nucleotide polymorphism (SNP) frequencies. Whites had a higher frequency of functioning alleles as compared with non-whites ( 92 vs. $74 \%, P=0.01$ ). The analysis was therefore adjusted for race to address racial differences in SNP frequencies. There were no associations between year of birth and frequency of high-risk alleles $(P=0.11)$. Analysis was also adjusted for TOF subtype, age at surgery, year of primary repair, type of repair, and duration of follow-up.

\section{HIF1A Genotype and RV Phenotype Associations}

A higher number of functioning HIF1A alleles was associated with lower RV outflow tract $z$-scores at last follow-up (estimated effect size (EST): $-0.365(0.118) z$ per functioning allele, $P=0.002$ ) (Figure 1a), slower progression of pulmonary valve regurgitation (EST: $+0.015 / \mathrm{y}^{2}$ for zero to three functioning alleles, $+0.009 / \mathrm{y}^{2}$ for four or five functioning alleles and $0.006 / \mathrm{y}^{2}$ for six functioning alleles, $\left.P=0.005\right)$, and slower progression of RV dilation over time (EST: $+0.021 / \mathrm{y}^{2}$ for zero to three functioning alleles, $+0.013 / \mathrm{y}^{2}$ for four or five functioning alleles and $+0.090 / \mathrm{y}^{2}$ for six functioning alleles, $P=0.02$ ) despite similar degrees of pulmonary regurgitation across genotypes early after repair $(P=0.26)$. At last follow-up, a higher number of functioning HIF1A alleles were also associated with a higher RV fractional area change (EST: $+1.2(0.6) \%$ per functioning allele, $P=0.04$ ) (Figure $1 \mathrm{~b}$ ), lower grade of tricuspid valve regurgitation (EST: $-0.066(0.037)$ per functioning allele, $P=0.07)$, and lower RV end-systolic area indexed to
Table 1. Patient characteristics

\begin{tabular}{|c|c|}
\hline Variable & Value \\
\hline Male gender & $99(55 \%)$ \\
\hline \multicolumn{2}{|l|}{ Race $^{a}$} \\
\hline Asian & $33(20 \%)$ \\
\hline Black & $9(6 \%)$ \\
\hline White & $111(69 \%)$ \\
\hline Other & $8(5 \%)$ \\
\hline \multicolumn{2}{|l|}{ Additional cardiac anomalies } \\
\hline Pulmonary artery anomalies & $35(19 \%)$ \\
\hline Patent ductus arteriosus & $27(15 \%)$ \\
\hline Atrial septal defect & $37(21 \%)$ \\
\hline Right aortic arch & $3(2 \%)$ \\
\hline Aorto-pulmonary collaterals & $7(4 \%)$ \\
\hline Coronary artery abnormalities & $6(3 \%)$ \\
\hline Other cardiac anomalies & $5(5 \%)$ \\
\hline Chromosome abnormalities/genetic syndrome & $15(8 \%)$ \\
\hline Systemic pulmonary arterial shunt before repair & $12(7 \%)$ \\
\hline \multicolumn{2}{|l|}{ Year of repair } \\
\hline 1991-1995 & $36(20 \%)$ \\
\hline 1996-2000 & $54(30 \%)$ \\
\hline $2001-2005$ & $37(21 \%)$ \\
\hline $2006-2009$ & $53(29 \%)$ \\
\hline Median age at repair (y, interquartile range) & $0.58(0.37-1.11)$ \\
\hline Neonatal repair ( $<2$ mo of age) & $11(6 \%)$ \\
\hline \multicolumn{2}{|l|}{ Type of repair } \\
\hline No patch repair & $66(37 \%)$ \\
\hline Transannular patch & $60(33 \%)$ \\
\hline Valve-sparing & $54(30 \%)$ \\
\hline $\mathrm{RV}$ reinterventions & $25(14 \%)$ \\
\hline Pulmonary valve replacement & $16(9 \%)$ \\
\hline Conduit replacement & $9(5 \%)$ \\
\hline
\end{tabular}

body size (EST: $-0.663(0.287) \mathrm{cm}^{3}$ per functioning allele, $P=$ $0.02)$. There was no association between number of functioning alleles and RV systolic pressure $(P=0.38)$ or degree of RV hypertrophy $(P=0.29)$. The overall freedom from $\mathrm{RV}$ reinterventions including pulmonary valve or conduit replacement was 92,84 , and $67 \%$ at 5,10 , and 15 y, respectively. Freedom from pulmonary valve reinterventions was significantly higher in patients with four or more functioning HIF1A alleles as compared with those with fewer than four functioning HIF1A alleles, with a decrease in hazard ratio for reinterventions by 0.77 for every additional functioning HIF1A allele $(P=0.04)$ (Figure 2).

\section{RV Myocardial Protein Expression and Fibrosis}

The expression levels of HIF1A ( $P=0.003)$, VEGF $(P=0.001)$, and TGF $\beta 1(P=0.001)$ were significantly higher in the RV of 

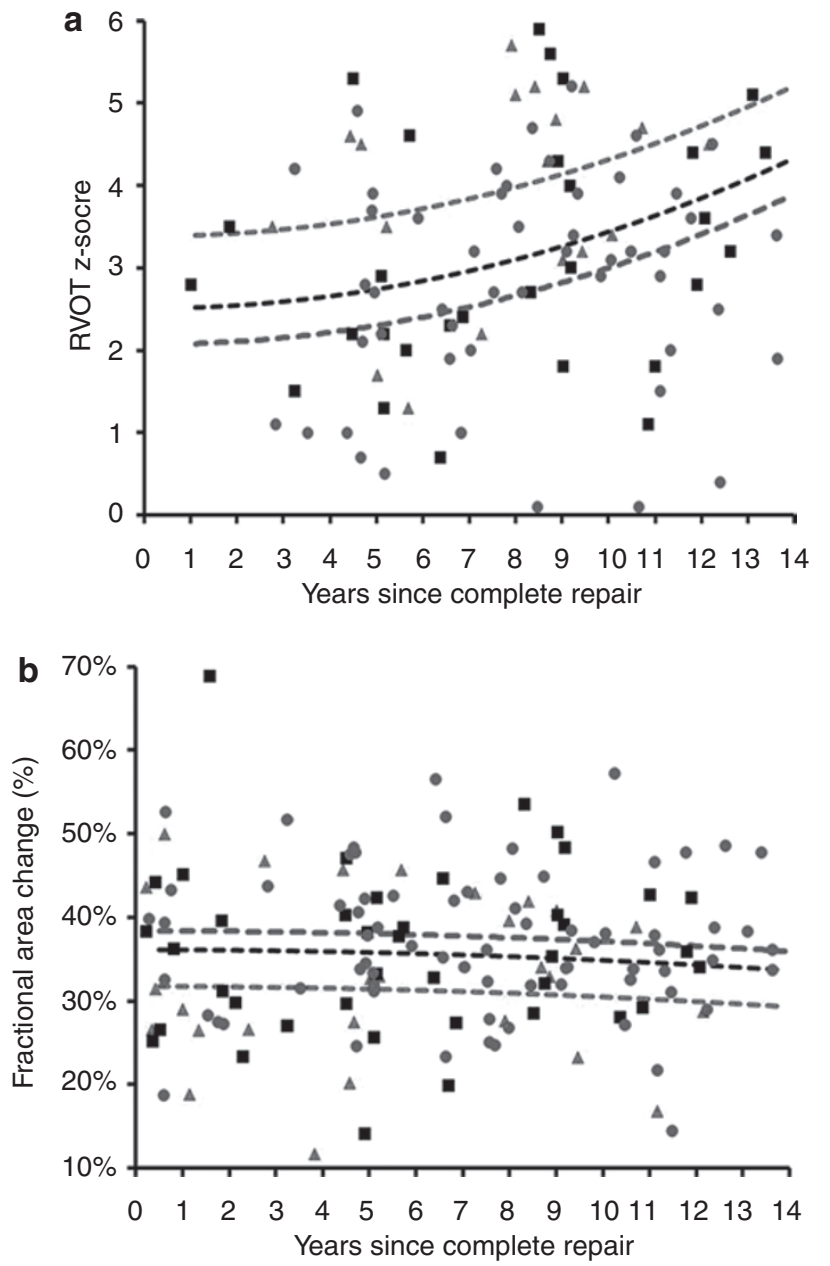

Figure 1. HIFA genotyping and RV remodeling. (a) HIF1A genotype and RVOT dilation: RVOT $z$-score increased progressively after TOF repair but was lower in patients with a higher number of functioning HIF1A alleles $(P=0.002)(n=180)$. (b) HIF1A genotype and RV function: RV systolic function assessed using fractional area of change decreased with time, but the rate of decline of systolic function was lower in TOF patients with a higher number of functioning HIF1A alleles $(P=0.04)$. Red triangles, one to three functioning HIF1A alleles; black squares, four or five functioning HIF1A alleles; blue circles, six functioning HIF1A alleles. The red, black, and blue lines are regression lines showing the relationship between RVOT $z$-score or fractional area change and number of functioning HIF1A alleles. HIF1A, hypoxia-inducible factor 1a; RVOT, right-ventricular outflow tract; TOF, tetralogy of Fallot.

patients undergoing TOF repair $(n=42$, median age $6 \mathrm{mo})$ as compared with patients undergoing ventricular septal defect repair $(n=20$, median age $5.3 \mathrm{mo}$ ) (Figure 3). Fibrosis area was also significantly greater in TOF patients as compared with controls $(P<0.001)$. Strong red nuclear staining indicated persistent nuclear localization of HIF1A in TOF, suggestive of chronic hypoxia. Representative images are shown in Figure 4.

We compared RV expression patterns in patients with four or more $(n=30$, median age $6 \mathrm{mo})$ vs. those with fewer than four functioning HIF1A alleles ( $n=12$, median age $6.4 \mathrm{mo}$ ). Patients with four or more functioning alleles had higher nuclear HIF1A expression $(P=0.001)$ and more fibrosis $(P=$ $0.02)$ as compared with patients with fewer than four functioning alleles (Figures 3 and 4). There was a direct correlation

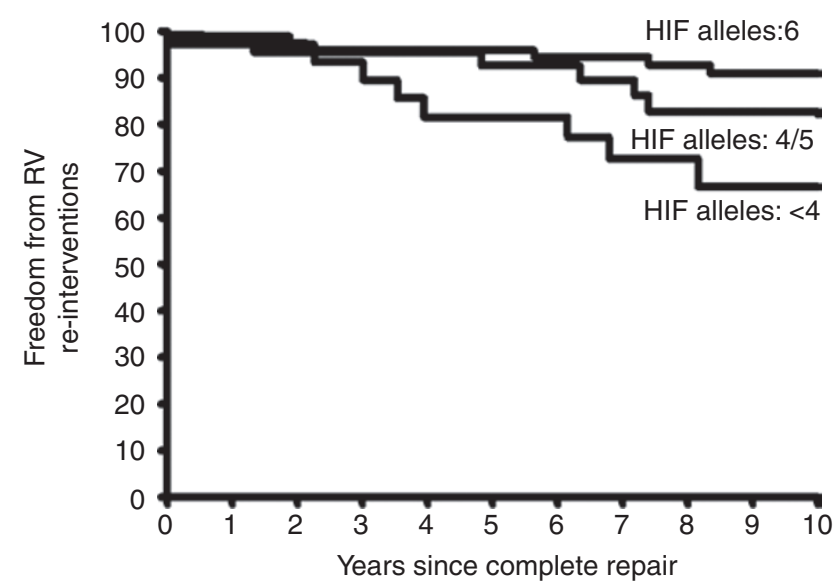

Figure 2. HIF1A genotype and freedom from RV reinterventions. Kaplan-Meier survival curve showing freedom from RV reinterventions (pulmonary valve or conduit replacement) during follow-up. Freedom from reinterventions was significantly lower in patients with four or more functioning HIF1A alleles as compared with those with fewer than four alleles. The hazard ratio for reintervention decreased by 0.78 for every additional functioning allele $(P=0.04)$. HIF1A, hypoxia-inducible factor $1 \mathrm{a}$; $\mathrm{HR}$, hazard ratio; $\mathrm{RV}$, right-ventricular.

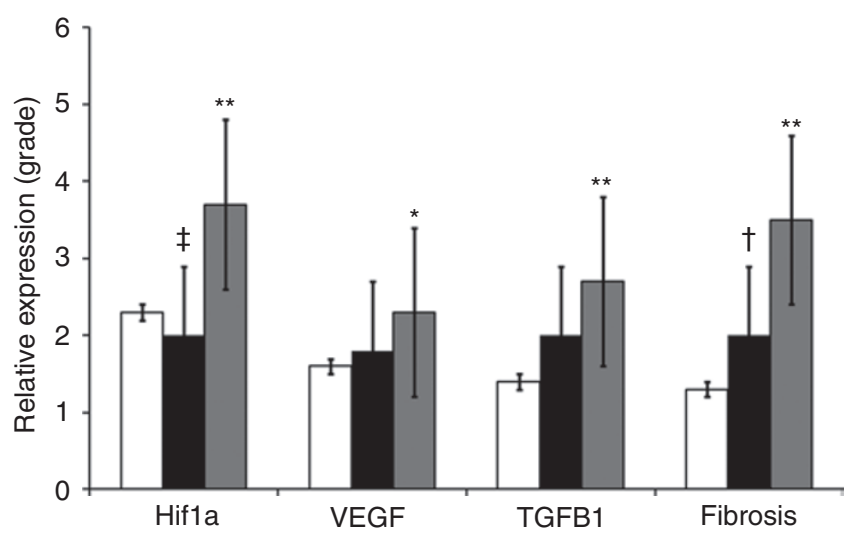

Figure 3. HIF1A genotype and RV protein expression. The relative intensity of protein expression in the RV before repair showed that HIF1A, VEGF, and TGF $\beta 1$ expression and fibrosis area were significantly higher in TOF patients with four or more functioning HIF1A alleles undergoing repair (gray) ( $n=42)$ as compared with ventricular septal defect controls undergoing repair (white) $(n=20)\left({ }^{*} P<0.01, * * P<0.001\right.$ vs. non-TOF controls). In the TOF cohort, HIF1A expression and fibrosis area were significantly higher in patients with four or more functioning HIF1A alleles (gray) as compared with patients with fewer than four alleles (black) ( $† P<0.05, \neq P<0.001$ vs. TOF with four or more functioning alleles). HIF1A, hypoxia-inducible factor $1 a$; RV, right-ventricular; TGFB1, transforming growth factor $\beta 1$; TOF, tetralogy of Fallot; VEGF, vascular endothelial growth factor.

between the number of functioning alleles and the intensity of nuclear HIF1A expression $\left(r^{2}=0.45, P=0.003\right)$ and the fibrosis area $\left(r^{2}=0.69, P=0.005\right)$. There was no difference in VEGF expression between genotype groups.

\section{Replication Cohort}

Sixty-nine additional eligible TOF patients were genotyped for replication analysis. The replication cohort was comparable to the primary cohort $-57 \%$ male, $68 \%$ white, $19 \%$ Asian, 

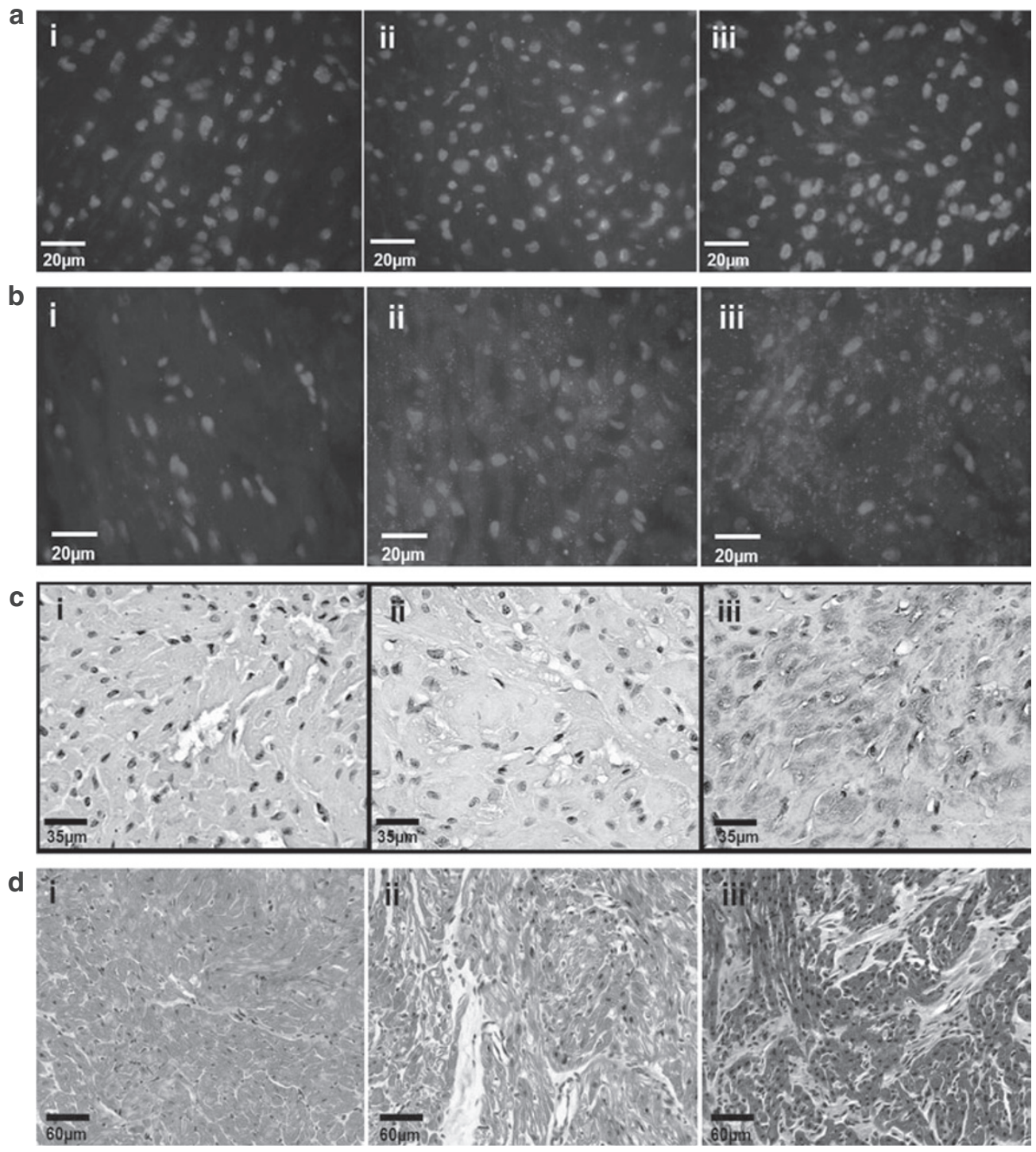

Figure 4. HIF1A genotype and RV histology. Representative images of RV immunostaining in RV from (i) unrepaired VSDs ( $n=20$ ), (ii) unrepaired TOF with fewer than four functioning HIF1A alleles $(n=12)$, and (iii) unrepaired TOF with four or more functioning alleles at complete repair $(n=30)$. DAPI represents blue nuclear staining. (a) Nuclear HIF1A expression (red) $(\times 600)$ was stronger in TOF patients with four or more functioning alleles (iii) as compared with controls (i) or TOF patients with fewer than four alleles (ii). (b) Cytoplasmic VEGF expression (red) ( $\times 600)$ was higher in TOF patients as compared with controls but was not significantly different between genotype groups. (c) Cytoplasmic TGF $\beta 1$ expression (brown) ( $\times 400)$ was higher in TOF patients with four or more functioning alleles (iii) as compared with controls (i). (d) RV myocardial fibrosis area (blue) ( $\times 200)$ was higher in TOF patients with four or more functioning alleles (iii) as compared with controls (i) or TOF patients with fewer than four alleles (ii). DAPI, 4',6-diamidino-2phenylindole; HIF1A, hypoxia-inducible factor $1 a$; RV, right-ventricular; TGF $\beta 1$, transforming-growth factor $\beta 1$; TOF, tetralogy of Fallot; VEGF, vascular endothelial growth factor; VSD, ventricular septal defect.

$7 \%$ black, and $6 \%$ other. Median age at repair was $0.58 \mathrm{y}$, and median age at enrollment was $5.4 \mathrm{y}$. Nineteen precent had an identifiable chromosomal anomaly or syndrome, $9 \%$ had a palliative shunt before complete repair, and $25 \%$ had a transannular patch repair. Seven patients (10\%) underwent pulmonary valve replacement, and four (6\%) underwent conduit replacement during follow-up. The frequencies of functioning HIF1A alleles in the replication cohort were comparable to those in the primary cohort: HIF1A145T (rs10873142) 74\%, HIF1A1326C (rs2057482) 83\%, and HIF1A1744C (rs11549465) 94\%. All genotypes were in Hardy-Weinberg equilibrium. Linear regression analysis comparing patients with homozygous
HIF1A functioning genotypes to heterozygous or nonfunctioning genotypes revealed a protective effect, with patients homozygous for HIF1A functioning genotypes demonstrating less RV dilation as measured by RV end-diastolic $z$-score (Figure 5). Also, patients with three or more functioning alleles had a lower frequency of RV dysfunction as compared with those with fewer than three functioning alleles ( 0 vs. $16 \%$, respectively, $P<0.05)$.

\section{DISCUSSION}

Our study identifies HIF1A signaling as an important determinant of myocardial adaptation to hypoxia before repair 


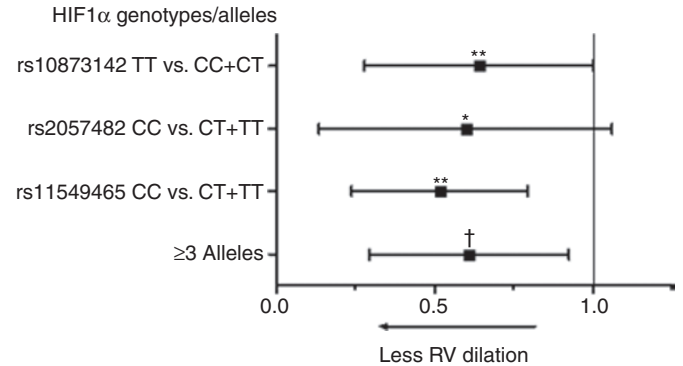

Figure 5. Replication cohort. The graph shows lower odds of right-ventricular (RV) dilation measured using RV end-diastolic $z$-score in patients homozygous for hypoxia-inducible factor 1a (HIF1A) functioning genotypes as compared with heterozygous or nonfunctioning genotypes. ${ }^{*} P=0.012$ for rs 2057482 (odds ratio (OR) 0.6, confidence interval (Cl) 0.14 $1.06) ;{ }^{* *} P=0.001$ for $\mathrm{rs} 10873142$ (OR $0.64, \mathrm{Cl} 0.28-0.99$ ), and rs 11549465 (OR 0.52, Cl 0.24-0.79). Patients with three or more functioning HIF1A alleles had lower odds of RV dilation as compared with those with fewer than three functioning alleles. $+P<0.001$ (OR 0.61, Cl 0.29-0.92) $(n=69)$.

and to RV remodeling during postsurgical follow-up. Genetic variation in the HIF1A gene accounted in part for the interindividual variability in this response. Patients with more HIF1A-functioning alleles had more RV fibrosis at the time of TOF repair but less progression of pulmonary insufficiency, less progression of RV dilation and dysfunction, and greater freedom from RV reinterventions after repair.

\section{HIF1A Genotype and Early RV Phenotype in Unrepaired TOF}

Until recently, evaluation of the causes of adverse RV remodeling after TOF repair have focused on anatomic and clinical factors, which have limited sensitivity in predicting which patients are likely to develop progressive RV dilation, dysfunction, and failure and need valve replacement. More recently, the emphasis has shifted to primordial factors that can affect the right ventricle before complete repair, i.e., during the fetal and neonatal periods. Of particular concern is the potential for ongoing hypoxia- and pressure overload-induced injury in the immature right ventricle while awaiting repair. This has led to the adoption of a neonatal repair strategy at several centers in an attempt to protect the RV during this vulnerable period, although the approach has had mixed results $(11,12)$. Identifying which patients may truly benefit from early repair has been challenging. Our data show that patients with a higher number of functioning HIF1A alleles had persistent nuclear HIF1A expression, upregulation of the profibrotic cytokine TGF $\beta 1$, and RV outflow tract fibrosis in the unrepaired state. The ability to correlate genotype with tissue expression from the same patient is a unique strength of this study and provides biologic support for the observed genetic association. One could argue, on the basis of these findings, that chronic hypoxia and/or mechanical stress in the unrepaired state can have an unfavorable impact on the RV phenotype, especially in patients with HIF1A-upregulation genotypes, and may provide a marker for patients at-risk for injury, who would benefit most from early repair. However, we need to be cautious and should not interpret all fibrosis as detrimental, given that some fibrosis is reversible and may in fact represent a temporary protective phenomenon during adverse environmental conditions such as hypoxia. The protective effect on RV remodeling and function on late follow-up indeed provides some support for the latter hypothesis.

\section{HIF1A Genotype and Late RV Phenotype in Repaired TOF}

The second important finding was cardioprotection in the form of slower progression of pulmonary insufficiency, less severe RV dilation on follow-up, and better preservation of RV systolic function in patients with a higher number of functioning HIF1A alleles in both the primary and replication cohorts. Not surprisingly, this was associated with greater freedom from RV reinterventions. Importantly, these associations persisted after correction for race, TOF subtype, age at repair, year of primary repair, type of repair, and duration of follow-up. Although, overall, this RV phenotype would be considered favorable because it delays the need for reinterventions, the susceptibility to fibrosis in the unrepaired state may suggest that the failure to dilate may be related to stiffer ventricles with more fibrosis. In a study of 92 adults, right and left ventricular fibrosis after TOF repair was common and was associated with ventricular dysfunction, exercise intolerance, and clinical arrhythmia $(13,14)$. Alternatively, HIF1A signaling may have a true cardioprotective effect through reversible fibroblast transformation of stressed cells during hypoxia in infancy that results in better long-term myocardial adaptation to hemodynamic load. Unfortunately, given the difficulties in detecting myocardial fibrosis noninvasively and the challenges in quantifying diastolic dysfunction by echocardiography, RV fibrosis and diastolic function were not serially assessed during follow-up. Recently, it has become possible to detect regional myocardial fibrosis noninvasively using cardiac magnetic resonance imaging (MRI) with late gadolinium enhancement $(13,14)$. A limitation of this technique, however, is that it does not detect early fibrosis, is qualitative, and relies on the difference in signal intensity between normal and scarred myocardium. Although this may be useful in evaluating regional fibrosis in patients with myocardial infarction, it appears to be less sensitive in patients with diffuse interstitial fibrosis $(15,16)$. New genetic markers or biomarkers that can identify at-risk patients earlier than current imaging modalities therefore hold promise as risk-stratification tools.

In summary, HIF1A is important in the myocardial response to hypoxia before repair and in preserving RV morphology and preventing progressive RV remodeling late after TOF repair. Knowledge of the HIF1A genotype may therefore prove useful in identifying patients who are at risk for progressive RV remodeling after repair and who may benefit from earlier pulmonary valve replacement before the onset of significant RV dilation. Whether this subset of patients may also benefit from early neonatal TOF repair to limit hypoxia-induced RV injury remains to be seen. Our study supports the need for additional genomic studies to identify susceptibility genes that can be used to identify at-risk patients early and intervene before RV remodeling and dysfunction become irreversible. 


\section{Limitations}

We did not assess diastolic dysfunction or severity of fibrosis during follow-up in this study. Although MRI imaging is emerging as an important noninvasive tool to assess fibrosis, its ability to detect early fibrosis is not known, as shown in a recent study of adult hypertrophic cardiomyopathy $(15,16)$. Also, we did not analyze cardiac MRI assessment of pulmonary regurgitant fraction and RV volumes because MRI data were available in only a subset of patients. Our study was not powered to analyze the independent association of myocardial fibrosis with late RV remodeling.

\section{Conclusion}

HIF1A genotype may provide a useful adjunct to clinical predictors to identify TOF patients at risk for progressive RV remodeling after TOF repair. Prospective validation of our findings is required. Overall, our findings highlight the importance of identifying genetic modifiers in biologic pathways of RV adaptation as a means of early risk stratification and use of genotype-guided optimization of type and timing of interventions.

\section{METHODS}

Patients with TOF $<18$ y old were prospectively enrolled in the SickKids Heart Centre Biobank Registry, an Ontario province-wide research network aimed at studying the genomic and environmental basis of heart defects (2008-2011). Patients with TOF who had undergone complete repair and were followed at our institution were studied as the primary cohort. Patients with history of TOF repair from other Ontario hospitals were included as a replication cohort. Clinical data were obtained from a review of the medical records. The study was approved by the Hospital for Sick Children Institutional Research Ethics Board, and informed consent was obtained from the parents/legal guardians.

\section{Genotyping Methods}

A blood or saliva sample was obtained for DNA extraction at enrollment in all eligible patients. SNP assays were performed using Applied Biosystems' Taqman SNP genotyping technology (Assays-by-Design) (Life Technologies, Burlington, Ontario, Canada) for the following three SNPs: HIF1A145C/T in the 5' untranslated region (rs10873142) (TTAAAACCCTAAATGTGACACAGTA[C/T]GCATGAGTGA TCATGCATCTCAAGA), HIF1A1326C/T (rs2057482)(CCTTTTTTT GGACACTGGTGGCTCA[C/T]TACCTAAAGCAGTCTATTT ATATTT), and HIF1A1744C/T in the $3^{\prime}$ untranslated region (rs11549465) (GTTACGTTCCTTCGATCAGTTGTCA[C/T]CATT AGAAAGCAGTTCCGCAAGCCC). "T", "C", and "C" alleles represent functioning alleles, whereas the single-nucleotide substitutions " $C$ ", "T", and " $\mathrm{T}$ " respectively represent loss-of-function alleles. These candidate SNPs were selected on the basis of previous association studies, known functional effects, allele frequency in the population, and prevalence in published medical literature (17). Loss-of-function SNPs have been associated with impaired adaptation to acute hypoxia or ischemia. However, the influence of these SNPs in chronic hypoxia is not known.

\section{Tissue Studies}

RV myocardial samples from the RV outflow tract were obtained at the time of complete repair from a subset of 42 patients with TOF and from 20 patients with isolated ventricular septal defects as controls. Immuno-histochemistry was performed on paraffin-embedded $5-\mu \mathrm{mol} / \mathrm{l}$ sections with standard techniques using the following primary antibodies: HIF1A, VEGF, and TGF $\beta 1$ (Abcam, Cambridge, MA) (18). Immunofluorescence was performed for VEGF and HIF1A using Texas red-conjugated antimouse immunoglobulin $\mathrm{G}$ as secondary antibody (Sigma, St Louis, MO). Sections were counterstained with 4',6-diamidino-2-phenylindole. Images were captured using a Nikon Eclipse E1000 microscope (×600 magnification) and Openlab 4.02 software (PerkinElmer, Waltham, MA). A horseradish peroxidase-conjugated streptavidin and di-amino benzidine method (Histostain Kit, Zymed Laboratories, Invitrogen, Carlsbad, CA) was used to evaluate TGF $\beta 1$ expression. Images were captured using a Leica DMLB2 microscope ( $\times 200$ magnification). HIF1A expression was quantified in eight fields per section using a GSA image analyzer (GSA Bansemer \& Scheel, Rostock, Germany)). The intensity of VEGF and TGF $\beta 1$ expression was semiquantitatively graded from 0 (none) to 4 (severe). Fibrosis area was evaluated on Masson's trichrome stain in six sections per sample ( $\times 200$ magnification).

\section{Echocardiographic Data}

Serial echocardiographic data were obtained after TOF repair as part of a standardized clinical protocol. A total of 1,046 studies were analyzed, with an average of six per patient and an average time from repair of $4.6 \mathrm{y}$ (maximum $16 \mathrm{y}$ ). Data were censored at the time of last follow-up or at pulmonary valve or conduit replacement. All patients underwent two-dimensional echocardiography using a standardized echocardiographic protocol used in the echocardiography laboratory in the Hospital for Sick Children (19). Data were stored on a clinical picture archiving and communication system (Syngo Dynamics, Siemens, Malvern, PA), and measurements were performed off-line by a single observer (A.J.). Echocardiographic analysis included qualitative assessment of the degree of pulmonary regurgitation, which was graded as trivial, mild, moderate, or severe on the basis of qualitative assessment. RV size was assessed using the RV outflow tract dimension and $z$-score as well as qualitatively with a grading of trivial, mild, moderate, or severe RV dilation. The RV dimensions were obtained by M-mode at the level of the mitral valve from a parasternal long-axis view. The $z$-scores were calculated on the basis of an institutional database of normal echocardiographic values. RV systolic function was assessed from the fractional area of change, which has been previously shown to correlate with MRI assessment of RV function (20). Fractional area change was derived from the RV end-diastolic area subtracted from the RV end-systolic area, divided by the RV end-diastolic area.

\section{Statistical Analysis}

Data are presented as means with SD, medians with minimum and maximum values, and frequencies as appropriate. Linear and logistic univariable regression models (adjusted for repeated measures with an autoregressive covariance structure for serial echocardiogram measurements) with maximum likelihood algorithms for parameter estimation were used to test for associations between genotypes and outcomes using an allelic model with a scale from 0 to 6 functioning alleles. All analyses were adjusted for race, TOF subtype, age at surgery, type of TOF repair, year of primary repair, and duration of follow-up (Table 1). The differences in outcomes between patients with and without risk alleles were reported as EST with 95\% confidence intervals for linear regression and hazard ratio with $95 \%$ confidence interval for logistic regression models. All models with time-dependent outcomes were adjusted for duration of follow-up. Natural logarithmic transformations were applied to variables with skewed distribution. Tissue results were compared between TOF and ventricular septal defect samples and in TOF patients with fewer than four vs. four or more functioning alleles using Student's $t$-test. All statistical analyses were performed using SAS v.9.2 (SAS Institute, Cary, NC).

\section{STATEMENT OF FINANCIAL SUPPORT}

This work was supported by the Heart and Stroke Foundation of Ontario, McLaughlin Centre, SickKids Labatt Family Heart Centre, and the Roma and Marvin Auerback Endowment Funds. There are no relationships with industry relevant to this work.

\section{ACKNOWLEDGMENTS}

We acknowledge the SickKids Heart Centre Biobank for access to DNA and tissue samples and the SickKids Centre for Applied Genomics for genotyping of candidate SNPs.

\section{REFERENCES}

1. Hoffman JI, Kaplan S, Liberthson RR. Prevalence of congenital heart disease. Am Heart J 2004;147:425-39.

2. Hickey EJ, Veldtman G, Bradley TJ, et al. Late risk of outcomes for adults with repaired tetralogy of Fallot from an inception cohort spanning four decades. Eur J Cardiothorac Surg 2009;35:156-64; discussion 164. 
3. Therrien J, Provost Y, Merchant N, Williams W, Colman J, Webb G. Optimal timing for pulmonary valve replacement in adults after tetralogy of Fallot repair. Am J Cardiol 2005;95:779-82.

4. Therrien J, Siu SC, McLaughlin PR, Liu PP, Williams WG, Webb GD. Pulmonary valve replacement in adults late after repair of tetralogy of fallot: are we operating too late? J Am Coll Cardiol 2000;36:1670-5.

5. Coats L, Khambadkone S, Derrick G, et al. Physiological consequences of percutaneous pulmonary valve implantation: the different behaviour of volume- and pressure-overloaded ventricles. Eur Heart J 2007;28:1886-93.

6. Semenza GL, Agani F, Feldser D, et al. Hypoxia, HIF-1, and the pathophysiology of common human diseases. Adv Exp Med Biol 2000;475:123-30.

7. Tekin D, Dursun AD, Xi L. Hypoxia inducible factor 1 (HIF-1) and cardioprotection. Acta Pharmacol Sin 2010;31:1085-94.

8. Sun S, Ning X, Zhang Y, et al. Hypoxia-inducible factor-1alpha induces Twist expression in tubular epithelial cells subjected to hypoxia, leading to epithelial-to-mesenchymal transition. Kidney Int 2009;75:1278-87.

9. Zeisberg EM, Tarnavski O, Zeisberg M, et al. Endothelial-to-mesenchymal transition contributes to cardiac fibrosis. Nat Med 2007;13:952-61.

10. Reddy S, Osorio JC, Duque AM, et al. Failure of right ventricular adaptation in children with tetralogy of Fallot. Circulation 2006;114(1 Suppl):I37-42.

11. Pigula FA, Khalil PN, Mayer JE, del Nido PJ, Jonas RA. Repair of tetralogy of Fallot in neonates and young infants. Circulation 1999;100(19 Suppl):II157-61.

12. Van Arsdell GS, Maharaj GS, Tom J, et al. What is the optimal age for repair of tetralogy of Fallot? Circulation 2000;102(19 Suppl 3):III $123-9$.
13. Wald RM, Haber I, Wald R, Valente AM, Powell AJ, Geva T. Effects of regional dysfunction and late gadolinium enhancement on global right ventricular function and exercise capacity in patients with repaired tetralogy of Fallot. Circulation 2009;119:1370-7.

14. Babu-Narayan SV, Kilner PJ, Li W, et al. Ventricular fibrosis suggested by cardiovascular magnetic resonance in adults with repaired tetralogy of fallot and its relationship to adverse markers of clinical outcome. Circulation 2006;113:405-13.

15. Broberg CS, Chugh SS, Conklin C, Sahn DJ, Jerosch-Herold M. Quantification of diffuse myocardial fibrosis and its association with myocardial dysfunction in congenital heart disease. Circ Cardiovasc Imaging 2010;3:727-34.

16. Ho CY, López B, Coelho-Filho OR, et al. Myocardial fibrosis as an early manifestation of hypertrophic cardiomyopathy. N Engl J Med 2010;363: 552-63.

17. Simonson TS, Yang Y, Huff CD, et al. Genetic evidence for high-altitude adaptation in Tibet. Science 2010;329:72-5.

18. Auerbach SR, Manlhiot C, Reddy S, et al. Recipient genotype is a predictor of allograft cytokine expression and outcomes after pediatric cardiac transplantation. J Am Coll Cardiol 2009;53:1909-17.

19. Mertens LL, Friedberg MK. Imaging the right ventricle-current state of the art. Nat Rev Cardiol 2010;7:551-63.

20. Anavekar NS, Skali H, Bourgoun M, et al. Usefulness of right ventricular fractional area change to predict death, heart failure, and stroke following myocardial infarction (from the VALIANT ECHO Study). Am J Cardiol 2008;101:607-12. 